\title{
Investigating extremely low resistance ohmic contacts to silicon carbide using a novel test structure
}

\author{
Yue Pan*a, Aaron M. Collins ${ }^{\mathrm{a}}$, Fahid Algahtani ${ }^{\mathrm{a}}$, Patrick W. Leech ${ }^{\mathrm{a}}$, Geoffrey K. Reeves ${ }^{\mathrm{a}}$, Philip \\ Tanner ${ }^{\mathrm{b}}$, Anthony S. Holland ${ }^{\mathrm{a}}$ \\ ${ }^{a}$ School of Electrical and Computer Engineering, RMIT University, Melbourne, Victoria 3001, \\ Australia; ${ }^{b}$ Queensland Microtechnology Facility, Griffith University, Brisbane, Australia
}

\begin{abstract}
Low resistance contracts to highly doped silicon carbide ( $\mathrm{SiC}$ ) are investigated. Using a novel test structure that is easy to fabricate and easy to use, this paper demonstrates how it is used to reliably determine relatively low specific contact resistivities which vary with heat treatment. The test structure requires no error correction and is not affected by parasitic resistances. Using the test structure, small changes in specific contact resistivity are determined for small temperature changes. Results will be presented and discussed on the application of this novel test structure for nickel to highly doped $\mathrm{SiC}$.
\end{abstract}

Keywords: Silicon carbide, contact resistance, ohmic contact, specific contact resistivity

\section{INTRODUCTION}

The compound semiconductor silicon carbide has excellent electrical and physical properties. Like all semiconductor devices, low resistance ohmic contacts to silicon carbide are required to minimise power losses in SiC devices. Specific contact resistivity $\left(\rho_{c}\right)$ is an extremely important parameter, which quantifies this metal to semiconductor ohmic contact. The measurement of $\rho_{c}$ for ohmic contacts to semiconductor has been reported by a number of people who used transmission line model and circular transmission line model ${ }^{1-7}$.

In this paper, we present a novel test structure for determining $\rho_{c}$ with experimental results for Ni to SiC contacts. Highly doped epitaxial SiC samples were each coated with $200 \mathrm{~nm}$ of nickel and patterned using a novel two-electrode ohmic contact test structure. Results obtained for sheet resistance of the active SiC layer agreed with Van der Pauw measurements and like the specific contact resistivity measurements, were consistent across many samples. Sheet resistance was $28 \Omega /$ sq. (independent of heat treatment) and the specific contact resistivity varied with varying heat treatments from low $8.3 \times 10^{-7} \Omega \cdot \mathrm{cm}^{2}$ to $5.6 \times 10^{-6} \Omega \cdot \mathrm{cm}^{2}$. The convenience and sensitivity of the test structure allowed for ease of determination of the appropriate heat treatment, to minimise the specific contact resistivity. Materials analysis was conducted to demonstrate the high crystal quality, low surface roughness, and appropriate stoichiometry of the SiC layer. The electrodes fabricated were exact in dimensions and these could be conveniently measured. The accurate measurement of electrode dimensions using a scanning electron microscope resulted in highly consistent results to be determined from electrical measurements using electrodes of different dimensions. Being circular electrodes allowed for accurate fabrication of such electrodes using contact lithography.

\section{THE MODEL}

The isotropic view of the proposed test structure is shown in Figure 1. It consists three pairs of patterns which have different radii of the central dot $r_{01}, r_{02}$ and $r_{03}$ from left to right. The inner and outer radii $r_{1}$ and $r_{1}$ of the outer electrode are the same for each pair. In this paper, the metal electrodes are assumed to be equalpotential.

As shown in figure 1, the total resistance $R_{T 1}, R_{T 2}$ and $R_{T 3}$ are measured between the central dot and the outer electrode for each pair of patterns.

*s3265073@student.rmit.edu.au; phone +61 415155585 


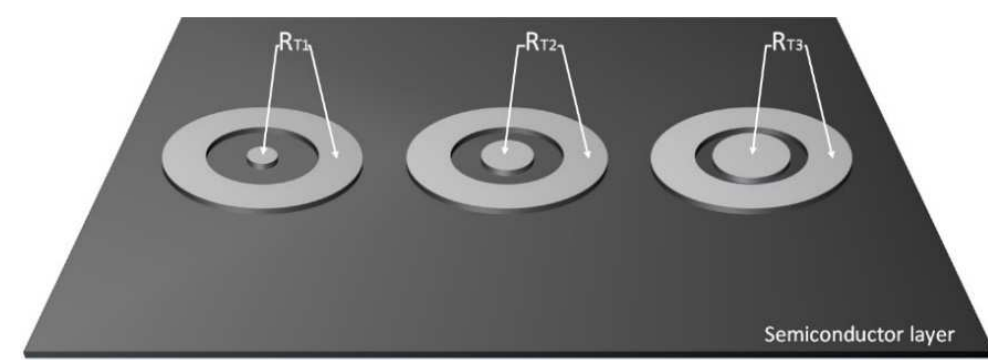

Figure 1. The isotropic view of the proposed test structure. The radii of the central dot from left to right are $r_{01}, r_{02}$ and $r_{03}$ respectively. The inner and outer radii for each pair of the patterns are the same which are $r_{1}^{\prime}$ and $r_{1}$.

As shown in equation (1), for each pair of the patterns, the total resistance $R_{T}$ consists of the contact resistance of the central dot $R_{C O}$, the parasitic resistance of the semiconductor ring $R_{P}$ and the contact resistance of the outer electrode $R_{C l}$.

$$
R_{T}=R_{C 0}+R_{P}+R_{C 1}
$$

As reported by Reeves ${ }^{8}$, the analytical equations for $R_{C 0}, R_{P}$ and $R_{C l}$ are

$$
\begin{gathered}
R_{C 0}=\frac{R_{S H}}{2 \pi \alpha r_{0}} \frac{I_{0}\left(\alpha r_{0}\right)}{I_{1}\left(\alpha r_{0}\right)} \\
R_{P}=\frac{R_{S H}}{2 \pi} \ln \left(\frac{r_{1}}{r_{0}}\right) \\
R_{C 1}=\frac{R_{S H}}{2 \pi \alpha r_{1}} \frac{\left[I_{1}\left(\alpha r_{1}^{\prime}\right) K_{0}\left(\alpha r_{1}\right)+I_{0}\left(\alpha r_{1}\right) K_{1}\left(\alpha r_{1}^{\prime}\right)\right]}{\left[I_{1}\left(\alpha r_{1}^{\prime}\right) K_{1}\left(\alpha r_{1}\right)-I_{1}\left(\alpha r_{1}\right) K_{1}\left(\alpha r_{1}^{\prime}\right)\right]}
\end{gathered}
$$

Hence, for these three pairs of the patterns, we have

$$
\begin{aligned}
& R_{T 1}=R_{C 01}+R_{P 1}+R_{C 1} \\
& R_{T 2}=R_{C 02}+R_{P 2}+R_{C 1} \\
& R_{T 3}=R_{C 03}+R_{P 3}+R_{C 1}
\end{aligned}
$$

Substitute equation (2)-(4) to (5)-(6), we have

$$
R_{T 1}-R_{T 2}=\frac{R_{S H}}{2 \pi}(F+\ln x)
$$

where $F=\frac{I_{0}\left(\alpha r_{01}\right)}{\alpha r_{01} I_{1}\left(\alpha r_{01}\right)}-\frac{I_{0}\left(\alpha r_{02}\right)}{\alpha r_{02} I_{1}\left(\alpha r_{02}\right)}$ and $x=\frac{r_{02}}{r_{01}}$

Also, we have

$$
R_{T 1}-R_{T 3}=\frac{R_{S H}}{2 \pi}\left(F^{\prime}+\ln y\right)
$$


where $F^{\prime}=\frac{I_{0}\left(\alpha r_{01}\right)}{\alpha r_{01} I_{1}\left(\alpha r_{01}\right)}-\frac{I_{0}\left(\alpha r_{03}\right)}{\alpha r_{03} I_{1}\left(\alpha r_{03}\right)}$ and $y=\frac{r_{03}}{r_{01}}$

Note that $\alpha$ is the inverse of the transfer length $L t$

$$
\alpha^{2}=\frac{R_{S H}}{\rho_{c}}
$$

Then we can get

$$
K=\frac{R_{T 1}-R_{T 3}}{R_{T 1}-R_{T 2}}=\frac{F^{\prime}+\ln y}{F+\ln x}
$$

Since $K$ can be determined experimentally and $x$ and $y$ can be determined by selecting appropriate $r_{01}, r_{02}$ and $r_{03}$, we can plot $K$ as a function of $\alpha r_{01}$ using equation (11). Also we can plot $\alpha r_{01}$ as a function of $F$ or $F$ using equation (8)-(9).

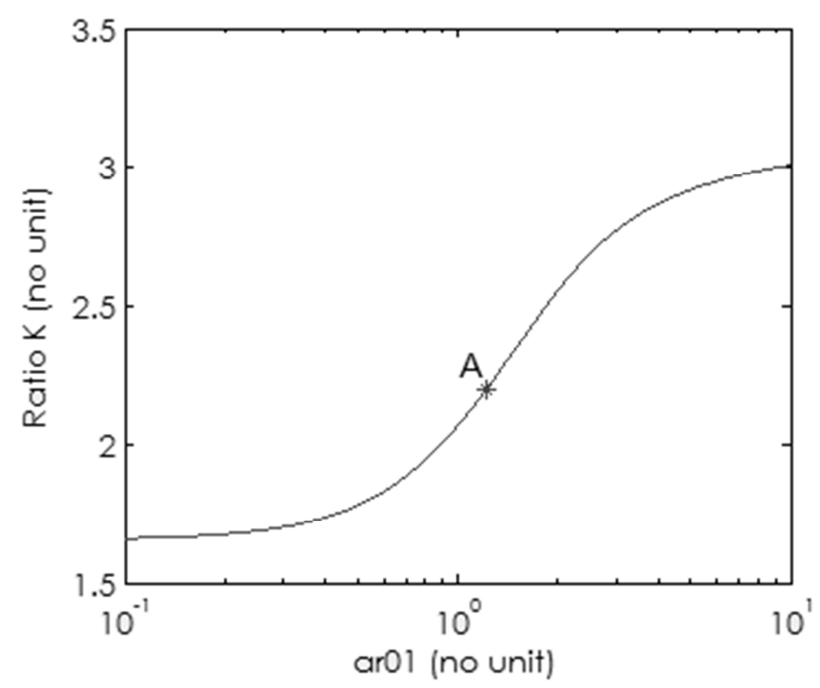

Figure 2. Ration $K$ versus $\alpha r 01$ for given rations of radii.

For experimental determined $K$, we can pick up a value for $\alpha r_{01}$ in figure 2. Point $A$ is an example data. Using this value in figure 3, we can get either $F$ or $F^{\prime}$. Points $A^{\prime}$ and $A^{\prime \prime}$ represent point $A$ in figure 2 . According to equation (8)-(9), we can get the sheet resistance $R_{S H}$. With known $R_{S H}$ and $\alpha$, using equation (10), specific contact resistivity $\rho_{c}$ can be found. 


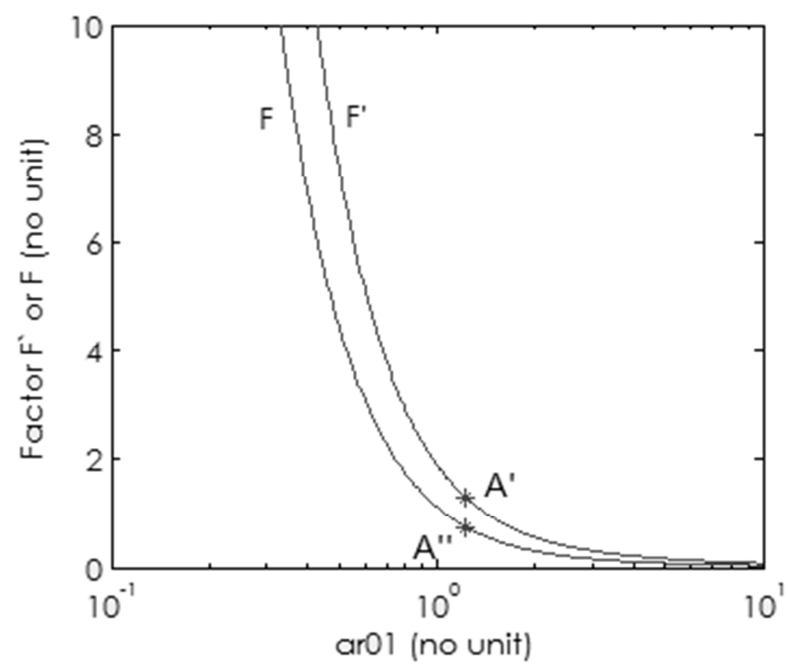

Figure 3. $F$ and $F$ ' versus $\alpha \mathrm{rO1}$ for given rations of radii.

\section{EXPERIMENTAL}

Experimental measurements were performed on $\mathrm{Ni}$ to $\mathrm{SiC}$ ohmic contacts. The $\mathrm{n}$-type $\mathrm{SiC}$ films were deposited to a thickness of $300 \mathrm{~nm}$ on $\mathrm{Si}$ substrate. The wafers were then cleaned using a sequence of rinses in acetone, isopropal alcohol and deionised water which were alternated with drying in a stream of nitrogen gas. A Ni layer with $200 \mathrm{~nm}$ thickness was then deposited on the SiC layer by electron beam evaporation. AZ 1521 photoresist was then spin coated over the samples followed by a soft baking at $95{ }^{\circ} \mathrm{C}$ for 90 seconds. The samples were then exposed to UV light for 10 seconds. After the photoresist was developed in 1: 4 AZ 400 for 15 seconds, the uncovered Ni was then removed by wet etching using a Ni etchant for 30 seconds at room temperature. The samples were then cleaned in acetone followed by IPA rinse and dried using nitrogen gas.

Figure 4 shows the optical micrographic of the proposed test structure fabricated on n-type SiC.

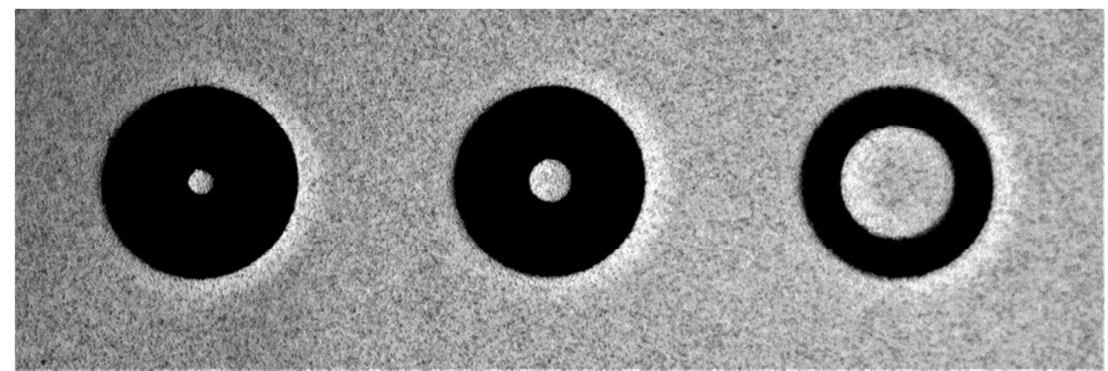

Figure 4. Optical micrographic of the proposed test structure fabricated on n-type SiC. The radii of the central dot from left to right are $5 \mu \mathrm{m}, 7.5 \mu \mathrm{m}$ and $17.5 \mu \mathrm{m}$ respectively.

\section{RESULTS AND DISCUSSION}

Current-voltage characteristics of as deposited $\mathrm{Ni}$ to $\mathrm{SiC}$ contacts are linear. Table 1 summarizes the results for the patterns where the dimensions vary from $r_{01}=5.0 \mu \mathrm{m}, r_{02}=7.5 \mu \mathrm{m}, r_{03}=17.5 \mu \mathrm{m}$ to $r_{01}=9.0 \mu \mathrm{m}, r_{02}=13.5 \mu \mathrm{m}, r_{03}=$ $31.5 \mu \mathrm{m}$. The measurements were performed using $1 \mathrm{~mA}$ current and two manipulators are used to keep the outer ring electrodes equalpotential. 
Table 1. Experimental results using the proposed test structure for determining the specific contact resistivity for as deposited Ni to SiC ohmic contacts

\begin{tabular}{|c|c|c|c|}
\hline Patterns & $\boldsymbol{\alpha r}_{\boldsymbol{O} \mathbf{1}}$ & $\boldsymbol{R}_{\boldsymbol{S H}}(\Omega / \mathrm{sq})$. & $\boldsymbol{\rho}_{\boldsymbol{c}}\left(\Omega \cdot \mathrm{cm}^{2}\right)$ \\
\hline 1 & 3.02 & 28.2 & $2.4 \times 10^{-6}$ \\
\hline 2 & 4.35 & 30.4 & $1.2 \times 10^{-6}$ \\
\hline 3 & 3.63 & 29.4 & $1.3 \times 10^{-6}$ \\
\hline 4 & 2.46 & 28.3 & $2.4 \times 10^{-6}$ \\
\hline 5 & 3.09 & 28.5 & $3.6 \times 10^{-6}$ \\
\hline 6 & 3.72 & 29.3 & $1.2 \times 10^{-6}$ \\
\hline 7 & 4.08 & 28.4 & $8.3 \times 10^{-7}$ \\
\hline 8 & 4.08 & 28.4 & $2.4 \times 10^{-6}$ \\
\hline 9 & 1.93 & 27.5 & $5.7 \times 10^{-6}$ \\
\hline 10 & 3.60 & 29.7 & $3.2 \times 10^{-6}$ \\
\hline
\end{tabular}

It is clearly that the value of $R_{S H}$ is approximately $28 \Omega$ /sq. which is very close to $27 \Omega /$ sq. that determined using Van der Pauw measurements. Specific contact resistivity varies from $8.3 \times 10^{-7} \Omega \cdot \mathrm{cm}^{2}$ to $5.7 \times 10^{-6} \Omega \cdot \mathrm{cm}^{2}$ which is highly acceptable and extremely low for metal to $\mathrm{SiC}$ ohmic contacts. This small variation also give the confidence in the values obtained by the proposed test structure.

\section{CONCLUSION}

Extremely low specific contact resistivity of as deposited Ni to SiC ohmic contacts were obtained by using a novel test structure which consists of a serious of circular electrode pairs. The sheet resistance was also determined by this test structure and it has been verified by Van der Pauw measurements. The lowest value of the specific contact resistivity obtained in the experiment is $8.3 \times 10^{-7} \Omega \cdot \mathrm{cm}^{2}$. The results have shown that for the appropriate geometries, this test structure is accurate and convenient for determine and since no error correction required and no complicated equations have to be solved.

\section{REFERENCES}

[1] D. K. Schroder, Semiconductor Material and Device Characterization, 3rd ed. Hoboken, NJ: Wiley, 2006, pp. 135157.

[2] Anthony S. Holland, Madhu Bhaskaran, Sharath Sriram, Geoffrey K. Reeves, Vykundh Ravichandran, Vishal D. Borase, Shreekkanth Bhaskaran, "Specific contact resistivity of Al-NiSi contacts using Cross Kelvin Resistor test structure chains,” SPIE Proceedings, vol. 6035, January 2006.

[3] Gong-Ru Lin, Jui L. Chang, "Transmission line measurement of gold contact on the arsenic-ion-implanted GaAs after rapid thermal annealing," SPIE Proceedings, vol. 4227, pp 29-34, October 2000.

[4] G. K. Reeves, H. B. Harrison, "Obtaining the specific contact resistance from transmission line model measurements," IEEE Electron Device Letters, vol. edl-3, no. 5, May 1982.

[5] Findlay K. W. J., Alexander W. J. C., Walton A. J., "The effect of contact geometry on the value of contact resistivity extracted from Kelvin structures,” proc. IEEE 1989 Int. Conference on Microelectronic Test Structures, vol 2, no. 1, March 1989.

[6] Hoki Kwon, Bong Koo Kim, Gyong Geun Park, Sung Woo Kim, Jaewan Choi, Jeong Soo Lee, Weon G. Jeong, "Ohmic characteristics of Pt and Ni/Au on Mg-doped $\mathrm{Al}_{\mathrm{x}} \mathrm{Ga}_{1-\mathrm{x}} \mathrm{N}$," SPIE Proceedings, vol. 6355, September 2006.

[7] Stephen J. Proctor, Loren W. Linholm, Jeffrey A. Mazer, "Direct measurements of interfacial contact resistance, end contact resistance, and interfacial contact layer uniformity," IEEE Transaction on Electronic Devices, vol. ed-30, no. 11, November, 1983.

[8] G. K. Reeves, "Specific contact resistance using a circular transmission line model," Solid State Electron, vol. 23, no. 5, pp. 487-490, May 1980. 